\title{
Goal-Directed Fluid Therapy Using Stroke Volume Variation Does Not Result in Pulmonary Fluid Overload in Thoracic Surgery Requiring One-Lung Ventilation
}

\author{
Sebastian Haas, ${ }^{1}$ Volker Eichhorn, ${ }^{2}$ Ted Hasbach, ${ }^{1}$ Constantin Trepte, ${ }^{1}$ Asad Kutup, ${ }^{3}$ \\ Alwin E. Goetz, ${ }^{1}$ and Daniel A. Reuter ${ }^{1}$ \\ ${ }^{1}$ Department of Anesthesiology, Center of Anesthesiology and Intensive Care Medicine, Cardiovascular Research Center, \\ University Medical Center Hamburg-Eppendorf, Martinistraße 52, 20246 Hamburg, Germany \\ ${ }^{2}$ Department of Anesthesiology and Intensive Care, Evangelisches Krankenhaus Mühlheim, 45468 Mühlheim, Germany \\ ${ }^{3}$ Department of General, Visceral and Thoracic Surgery, University Medical Center Hamburg-Eppendorf, 20246 Hamburg, Germany
}

Correspondence should be addressed to Sebastian Haas, shaas@uke.de

Received 1 April 2012; Accepted 8 May 2012

Academic Editor: Lars J. Bjertnaes

Copyright (C) 2012 Sebastian Haas et al. This is an open access article distributed under the Creative Commons Attribution License, which permits unrestricted use, distribution, and reproduction in any medium, provided the original work is properly cited.

\begin{abstract}
Background. Goal-directed fluid therapy (GDT) guided by functional parameters of preload, such as stroke volume variation (SVV), seems to optimize hemodynamics and possibly improves clinical outcome. However, this strategy is believed to be rather fluid aggressive, and, furthermore, during surgery requiring thoracotomy, the ability of SVV to predict volume responsiveness has raised some controversy. So far it is not known whether GDT is associated with pulmonary fluid overload and a deleterious reduction in pulmonary function in thoracic surgery requiring one-lung-ventilation (OLV). Therefore, we assessed the perioperative course of extravascular lung water index (EVLWI) and $\mathrm{p}_{\mathrm{a}} \mathrm{O}_{2} / \mathrm{F}_{\mathrm{i}} \mathrm{O}_{2}$-ratio during and after thoracic surgery requiring lateral thoracotomy and OLV to evaluate the hypothesis that fluid therapy guided by SVV results in pulmonary fluid overload. Methods. A total of 27 patients (group T) were enrolled in this prospective study with 11 patients undergoing lung surgery (group L) and 16 patients undergoing esophagectomy (group E). Goal-directed fluid management was guided by SVV (SVV < $10 \%$ ). Measurements were performed directly after induction of anesthesia (baseline—BL), 15 minutes after implementation OLV (OLVimpl15), and 15 minutes after termination of OLV (OLVterm15). In addition, postoperative measurements were performed at 6 (6postop), 12 (12postop), and 24 (24postop) hours after surgery. EVLWI was measured at all predefined steps. The $\mathrm{p}_{\mathrm{a}} \mathrm{O}_{2} / \mathrm{F}_{\mathrm{i}} \mathrm{O}_{2}-$ ratio was determined at each point during mechanical ventilation (group L: BL-OLVterm15; group E: BL-24postop). Results. In all patients (group T), there was no significant change $(P>0.05)$ in EVLWI during the observation period (BL: 7.8 \pm 2.5 , 24postop: $8.1 \pm 2.4 \mathrm{~mL} / \mathrm{kg}$ ). A subgroup analysis for group $\mathrm{L}$ and group $\mathrm{E}$ also did not reveal significant changes of EVLWI. The $\mathrm{p}_{\mathrm{a}} \mathrm{O}_{2} / \mathrm{F}_{\mathrm{i}} \mathrm{O}_{2}-$ ratio decreased significantly during the observation period (group L: BL: $462 \pm 140$, OLVterm15: $338 \pm 112$ mmHg; group E: BL: $389 \pm 101$, 24postop: $303 \pm 74 \mathrm{mmHg}$ ) but remained $>300 \mathrm{mmHg}$ except during OLV. Conclusions. SVV-guided fluid management in thoracic surgery requiring lateral thoracotomy and one-lung ventilation does not result in pulmonary fluid overload. Although oxygenation was reduced, pulmonary function remained within a clinically acceptable range.
\end{abstract}

\section{Introduction}

Early, preemptive strategies of hemodynamic optimization are an important factor for sufficient organ microcirculation and are considered to be associated with reduced morbidity and mortality [1]. Within this context, improvement of intravascular volume status seems essential. After central venous pressure (CVP) was identified as inappropriate for the assessment of intravascular volume status, volumetric and functional parameters of preload came into the focus of interest. Clinical studies were thus initiated to evaluate the potential of fluid management guided by these parameters. Although one study failed to demonstrate improvements, other studies have shown improvements in hemodynamics 
leading to benefits in clinical outcome when goal-directed fluid therapy was guided by global enddiastolic volume (GEDV), pulse pressure variation (PPV), or stroke volume variation (SVV) $[2-5]$. However, in thoracic surgery, the potential benefit or even disadvantage of volume management guided by functional parameters of preload have not been evaluated thus far. One possible disadvantage may be the fact that fluid management guided by functional parameters of preload is suspected to be a rather fluid aggressive approach since in the clinical studies the goal-directed group received more fluids than the control group [2-5]. Lopes and colleagues reported a more than double amount of fluid administration in the PPV-guided fluid management group [4]. It hast to be kept in mind that pulmonary fluid overload has been identified as an independent risk factor for the development of perioperative acute lung injury after lung surgery [6]. Therefore, a volume restrictive regime is usually recommended for lung surgery [6-9].

The extravascular fluid content of the lungs can be quantified using transpulmonary thermodilution (TCPTD) by measuring the extravascular lung water index (EVLWI). EVLWI by thermodilution was identified as more sensitive for quantifying the fluid content of the lungs than chest radiographs [10-12]. Furthermore, EVLWI is known as a prognostic parameter for clinical outcome in critically ill patients [13-17].

Fluid management in thoracic surgery is of particular importance because of the influence of one-lung ventilation $(\mathrm{OLV})$. It has been reported that OLV by itself can be a cause of postoperative pulmonary edema [17-20]. Edema formation after OLV is explained by oxidative stress during and in particular immediately following-OLV by reexpansion of the deflated lung, once conventional ventilation is reestablished [19-22]. Therefore, OLV might act as an additional factor for aggravating a perioperative pulmonary fluid overload.

In open chest conditions, the use of functional parameters of preload such as SVV to predict volume responsiveness is controversial [23-28]. As this question is not conclusively answered, the feasibility of SVV-guided fluid management remains unclear.

The hypothesis of our study was that fluid management guided by SVV results in fluid overload of the lungs during and after thoracic surgery that requires lateral thoracotomy and OLV. Therefore, the aim of the study was to investigate the influence of SVV-guided fluid management on the perioperative course of the formation of pulmonary extravascular fluid content as measured by EVLWI (first endpoint) and gas exchange measured by $\mathrm{p}_{\mathrm{a}} \mathrm{O}_{2} / \mathrm{F}_{\mathrm{i}} \mathrm{O}_{2}$-ratio (second endpoint) in thoracic surgery requiring lateral thoracotomy and OLV. Furthermore, 30-day mortality was assessed.

\section{Materials and Methods}

Approval for this study was provided by the Ethics Committee of the Hamburg Medical Board (Aerztekammer Hamburg). All patients gave written informed consent.
2.1. Patients. A total of 27 patients (group T, $n=27$ ) scheduled for elective thoracic surgery requiring OLV were enrolled in this prospective study. Exclusion criteria were age under 18 years, cardiac arrhythmias and/or atrial fibrillation, and the presence of contraindications to femoral arterial catheterization.

2.2. Anesthesia. All patients were premedicated with midazolam $0.1 \mathrm{mg} / \mathrm{kg}$ orally before arriving in the operating room. All patients received an epidural catheter at level Th4 to Th7. A bolus of $0.125 \mathrm{~mL} / \mathrm{kg}$ of $0.5 \%$ bupivacaine and $10 \mu \mathrm{g}$ sufentanil was administered followed by a continuous administration of $0.5 \%$ bupivacaine $(0.05 \mathrm{~mL} / \mathrm{kg} / \mathrm{h})$. After surgery, administration of bupivacaine was stopped and $0.2 \%$ ropivacaine was administered at an infusion rate of $8 \mathrm{~mL} / \mathrm{h}$ and was adjusted to the clinical situation. Directly after placement of the epidural catheter, general anesthesia was induced with $0.7 \mu \mathrm{g} / \mathrm{kg}$ sufentanil, $2.5 \mathrm{mg} / \mathrm{kg}$ propofol, and $0.6-0.8 \mathrm{mg} / \mathrm{kg}$ rocuronium. Anesthesia was maintained with isoflurane 1$1.5 \%$ in oxygen and sufentanil, and rocuronium was used for further relaxation. During surgery, a dosage of 0.2$0.3 \mu \mathrm{g} / \mathrm{kg}$ sufentanil was administered every 45 minutes or when clinically required. Neuromuscular monitoring was used for further rocuronium application, and a dosage of $0.1-0.2 \mathrm{mg} / \mathrm{kg}$ rocuronium was repeated when the train of four (TOF) ratio was $>0.5$. A left-sided double-lumen tube (Broncho-Cath; 37-41 French, Mallinckrodt Medical Ltd, Ireland) was introduced and adjusted using a fiberoptic bronchoscope.

2.3. Ventilation. Ventilation was performed using pressurecontrolled mode. During conventional ventilation of both lungs, pressure control was adjusted to achieve tidal volumes of $8 \mathrm{~mL} / \mathrm{kg}$. A positive end-expiratory pressure (PEEP) of $8 \mathrm{~cm} \mathrm{H}_{2} \mathrm{O}$ was selected. The inspiratory-expiratory ratio was $1: 1.7$. The respiratory rate was adjusted to maintain the arterial partial pressure of carbon dioxide between 36 and $44 \mathrm{mmHg}$. During OLV, the ventilation pattern was modified using a PEEP of $3 \mathrm{~cm} \mathrm{H}_{2} \mathrm{O}$, and tidal volumes of $4-6 \mathrm{~mL} / \mathrm{kg}$. The inspired oxygen fraction was initially 1.0 and decreased to a level which allowed maintenance of $\mathrm{P}_{\mathrm{a}} \mathrm{O}_{2}>80 \mathrm{mmHg}$.

2.4. Hemodynamic Monitoring. A central venous line was placed into the internal jugular vein for the continuous monitoring of central venous pressure, drug administration and injection of cold indicator for thermodilution. A 5Fr thermistor-tipped catheter (PICCO, PV2025L20, Pulsion Medical Systems AG, Munich, Germany) was inserted into the femoral artery and connected to a hemodynamic monitor (PiCCO plus, Pulsion Medical Systems AG, Munich, Germany) for continuous measurement of SVV, arterial pressure and intermittent assessment of cardiac index (CI), global enddiastolic volume index (GEDI), stroke volume index (SVI), and EVLWI by TCPTD. Thermodilution measurements were performed by three sequential central venous injections of $10 \mathrm{~mL}$ cold saline solution $\left(<8^{\circ} \mathrm{C}\right)$. All thermodilution curves were examined, and measurements were 
accepted if none of the three consecutive values differed by more than $10 \%$ from the mean.

2.5. Study Protocol. All patients received continuous infusion of crystalloid infusion (Sterofundin Ecoflac Plus, Braun AG, Melsung Germany) at a rate of $9 \mathrm{~mL} / \mathrm{kg} / \mathrm{h}$ intraoperatively. Continuous administration was reduced to $4 \mathrm{~mL} / \mathrm{kg} / \mathrm{h}$ after surgery and continuous administration was further reduced to $2 \mathrm{~mL} / \mathrm{kg} / \mathrm{h}$ after extubation. These rather high infusion rates were chosen to provide a rather fluid aggressive study protocol. $1000 \mathrm{~mL}$ Sterofundin content $5.5 \mathrm{~g} \mathrm{NaCl}, 0.3 \mathrm{~g}$ $\mathrm{KCl}, 0.37 \mathrm{~g} \mathrm{CaCl}_{2}, 0.2 \mathrm{~g} \mathrm{MgCl}_{2}$, and $0.05 \mathrm{~g}$ natrium-lactate. Osmolarity is $299 \mathrm{mOsm} / \mathrm{L}$. Additionally, a bolus of $5 \mathrm{~mL} / \mathrm{kg}$ colloid (Voluven 130/0.4 6\%, Fresenius Kabi AG, Bad Homburg, Germany) was given when SVV was above $10 \%$ and repeated if necessary until SVV returned to below $10 \%$. Colloid administration, in order to achieve an SVV of lower than $10 \%$ was primarily done prior to open-chest conditions and continued during the surgical phase with a laterally opened thoracic cavity and after surgery. If clinically indicated (according to the International Normalized Ratio (INR) > 1.6 in combination with active bleeding), the required fluid loading was done with fresh frozen plasma. When mean arterial pressure dropped below $60 \mathrm{mmHg}$ despite fluid resuscitation or during sudden blood loss, continuous norepinephrine administration was initiated. After extubation, SVV-guided fluid management was discontinued because SVV is not validated for use during spontaneous breathing. Hemodynamic measurements as well as arterial blood gas analyses were performed after induction of anesthesia (baseline-BL), 20 min after implementation of OLV (OLVimpl15) and $15 \mathrm{~min}$ after termination of OLV (OLVterm15), as well as at $6 \mathrm{~h}$ (6postop), $12 \mathrm{~h}$ (12postop), and $24 \mathrm{~h}$ (24postop) after the end of surgery.

2.6. Surgical Procedures. In the 11 patients with lung surgery, lobectomy was performed in 9 patients and bi-lobectomy in 2 patients. In the esophageal surgery group all 16 patients underwent transthoracic esophagectomy with two-field lymphadenectomy and reconstruction achieved by gastric pull up. None of the patients received neoadjuvant treatment.

2.7. Extravascular Lung Water Index (EVLWI) and Pulmonary Function. EVLWI was measured by transpulmonary thermodilution for quantification of pulmonary extravascular fluid content. To evaluate pulmonary compliance, the static pulmonary compliance $\mathrm{C}\left[\mathrm{L} / \mathrm{cmH}_{2} \mathrm{O}\right]$ was assessed using the equation $C=V / \Delta P$, where $V$ is the tidal volume and $\triangle P$ is the difference of inspiration pressure and PEEP. Oxygenation was quantified by calculating the $\mathrm{p}_{\mathrm{a}} \mathrm{O}_{2} / \mathrm{F}_{\mathrm{i}} \mathrm{O}_{2}$-ratio (arterial partial pressure of oxygen $\mathrm{p}_{\mathrm{a}} \mathrm{O}_{2}$ /inspiratory oxygen concentration $\mathrm{F}_{\mathrm{i}} \mathrm{O}_{2}$ ) for each patient at each point of measurement as long as the patient was intubated and mechanically ventilated (Group L: from BL to OLVterm15, Group E: from BL to 24postop).

2.8. Metabolic and Hemodynamic Parameters. Blood lactate, central venous oxygen saturation $\left(\mathrm{ScvO}_{2}\right)$, and base excess (BE) were assessed. Furthermore, hemodynamic data such as mean arterial pressure $\left(\mathrm{AP}_{\text {mean }}\right)$, central venous pressure (CVP), global enddiastolic volume index (GEDI), stroke volume index (SVI), cardiac index (CI), pulmonary compliance, and norepinephrine administration were recorded at the predefined steps BL-24postop to describe metabolic and hemodynamic consequences during and after SVVguided fluid management in thoracic surgery requiring lateral thoracotomy and OLV.

2.9. Subgroup Analysis. To further evaluate our hypothesis, a subgroup analysis of two subgroups was performed. Firstly, the course of EVLWI was explored in the lung surgery (group L: $n=11$ ). Lung surgery is usually associated with a relatively short period of OLV and direct trauma to the lungs. Secondly, the course of EVLWI was investigated in transthoracic esophagectomy (group E: $n=16$ ), an intervention involving severe general surgical trauma, a longer period of OLV, and higher fluid turnover.

2.10. Statistical Analysis. Descriptive statistical analysis was performed using SigmaStat and SigmaPlot (Systat Software, Inc., Germany). Student $t$-test was performed for corresponding group comparison regarding patients characteristics and surgery data between group L and group E. Normally distributed data (Kolmogorov-Smirnov-Test) were analyzed with a Tukey's one-way analysis of variance for repeated measurements (ANOVA), nonnormally distributed parameters were analyzed with Kruskal-Wallis Analysis of Variance (ANOVA) on Ranks. Results are given as mean \pm standard deviation (SD). A $P$ value $<0.05$ was considered statistically significant.

\section{Results}

Patient's characteristics and comorbidities are given in Table 1. Patients did not differ significantly regarding age, body mass index, and ASA classification. Data regarding surgery, fluid administration and diuresis are presented in Table 2. All parameters were significantly higher in group $\mathrm{E}$ than in group $\mathrm{L}(P<0.05)$ apart from the duration of OLV $(P=0.057)$ and red blood cell administration $(P=0.223)$.

3.1. Extravascular Lung Water Index (EVLWI). In all patients (group T), EVLWI did not change significantly during the observation period (BL: $7.8 \pm 2.5,24$ postop: $8.1 \pm$ $2.4 \mathrm{~mL} / \mathrm{kg}$ ). The course of EVLWI in the subgroup analysis (group L: BL: $7.9 \pm 1.7 \mathrm{~mL} \times \mathrm{kg}^{-1}$, 24postop: $7.2 \pm$ $1.9 \mathrm{~mL} / \mathrm{kg}$; group E: BL: $7.8 \pm 3 \mathrm{~mL} / \mathrm{kg}, 24$ postop: $9.1 \pm$ $2.5 \mathrm{~mL} / \mathrm{kg}$ ) also revealed no significant changes. The highest mean of EVLWI was measured in group $\mathrm{E}$ at 24postop $(9.1 \mathrm{~mL} / \mathrm{kg})$.

3.2. $\mathrm{P}_{a} \mathrm{O}_{2} / \mathrm{F}_{i} \mathrm{O}_{2}$-Ratio. In all patients (group $\mathrm{T}$ ), the $\mathrm{p}_{\mathrm{a}} \mathrm{O}_{2} /$ $\mathrm{F}_{\mathrm{i}} \mathrm{O}_{2}$-ratio decreased when comparing values prior to (BL) and after OLV (OLVterm15) (BL: $419 \pm 122 \mathrm{mmHg}$, OLVterm15: $334 \pm 92 \mathrm{mmHg}$ ). In subgroup $\mathrm{L}$, the $\mathrm{p}_{\mathrm{a}} \mathrm{O}_{2} / \mathrm{F}_{\mathrm{i}} \mathrm{O}_{2}-$ ratio also decreased significantly from $462 \pm 140 \mathrm{mmHg}$ at $\mathrm{BL}$ to $338 \pm 112 \mathrm{mmHg}$ at OLVterm15. In group E, a decrease 
TABle 1: Patient's characteristics and comorbidities. Patients did not differ significantly $(P>0.05)$ between group L and group E regarding age, body mass index, and ASA classification.

\begin{tabular}{|c|c|c|c|c|}
\hline & All patients $(n=27)$ & Group L $(n=11)$ & Group $\mathrm{E}(n=16)$ & $P$ value \\
\hline Age [years] & $61.3 \pm 11.6$ & $62.1 \pm 10.6$ & $60.4 \pm 13.4$ & $P=0.72$ \\
\hline Body mass index $\left[\mathrm{kg} / \mathrm{m}^{2}\right]$ & $25.4 \pm 5.2$ & $24.7 \pm 5.5$ & $26 \pm 5.2$ & $P=0.56$ \\
\hline ASA classification & $2.7 \pm 0.3$ & $2.5 \pm 0.3$ & $2.8 \pm 0.3$ & $P=0.65$ \\
\hline Coronary artery disease & 5 & 2 & 3 & \\
\hline Impaired ventricular function $(\mathrm{EF}<40 \%)$ & 3 & 1 & 2 & \\
\hline Renal insufficiency & 4 & 2 & 2 & \\
\hline Chronic obstructive pulmonary disease & 10 & 8 & 2 & \\
\hline
\end{tabular}

TABLE 2: Surgical data, fluid administration, and diuresis. *Statistical significance between group L and group E analyzed by students $t$-test $(P<0.05)$.

\begin{tabular}{|c|c|c|c|c|}
\hline & All patients $(n=27)$ & Group L $(n=11)$ & Group $\mathrm{E}(n=16)$ & $P$ value \\
\hline Duration of surgery [min] & $294.3 \pm 144.4$ & $177.5 \pm 76.7$ & $375.7 \pm 123.6^{*}$ & $P<0.001$ \\
\hline Duration of OLV [min] & $134.8 \pm 25.6$ & $113.3 \pm 55.7$ & $149.3 \pm 39.3$ & $P=0.057$ \\
\hline Blood loss during surgery $[\mathrm{mL}]$ & $538 \pm 784$ & $190 \pm 347$ & $778 \pm 914$ & $P=0.037$ \\
\hline Number of patients received noradrenaline & 22 & 6 & 16 & \\
\hline Crystalloid administered during observation period $[\mathrm{mL} / \mathrm{kg} / \mathrm{h}]$ & $3.4 \pm 0.8$ & $2.8 \pm 0.8$ & $3.8 \pm 0.8^{*}$ & $P=0.003$ \\
\hline Colloid administered during observation period [mL/kg/h] & $1.2 \pm 0.4$ & $0.6 \pm 0.3$ & $1.5 \pm 0.5^{*}$ & $P<0.001$ \\
\hline Fresh frozen plasma administered during observation period $[\mathrm{mL}]$ & $514 \pm 840$ & 0 & $770 \pm 933^{*}$ & $P=0.012$ \\
\hline Packed red blood cells administered during observation period $[\mathrm{mL}]$ & $545 \pm 697$ & $320 \pm 345.1$ & $640 \pm 642$ & $P=0.223$ \\
\hline Diuresis $[\mathrm{mL} / \mathrm{kg} / \mathrm{h}]$ & $1.3 \pm 0.4$ & $0.9 \pm 0.3$ & $1.6 \pm 0.4^{*}$ & $P<0.001$ \\
\hline
\end{tabular}

in the $\mathrm{p}_{\mathrm{a}} \mathrm{O}_{2} / \mathrm{F}_{\mathrm{i}} \mathrm{O}_{2}$-ratio was observed 24 hrs after surgery (BL $389 \pm 101 \mathrm{mmHg}, 24$ postop: $303 \pm 74 \mathrm{mmHg}$ ). The lowest mean $\mathrm{p}_{\mathrm{a}} \mathrm{O}_{2} / \mathrm{F}_{\mathrm{i}} \mathrm{O}_{2}$-ratio was observed in group E except for during OLV at timepoint 24postop $(303 \pm 74 \mathrm{mmHg})$. Patients of group L were extubated immediately after the end of surgery, whereas patients of group E were extubated $24 \mathrm{~h}$ after the end of surgery.

3.3. Cardiac Index. CI was increased at timepoints OLVimpl15 and 6postop compared to baseline timepoint BL in all patients. (BL: $2.8 \pm 0.9 \mathrm{~L} / \mathrm{min} / \mathrm{m}^{2}$, OLVimpl15: $3.9 \pm$ $0.9 \mathrm{~L} / \mathrm{min} / \mathrm{m}^{2}$, 6postop: $\left.3.5 \pm 0.9 \mathrm{~L} / \mathrm{min} / \mathrm{m}^{2}\right)$. In the subgroup analysis, CI increased significantly at OLVimpl15 in group $\mathrm{E}\left(\mathrm{BL}: 2.7 \pm 0.9 \mathrm{~L} / \mathrm{min} / \mathrm{m}^{2}\right.$, OLVimpl15: $3.7 \pm$ $\left.1 \mathrm{~L} / \mathrm{min} / \mathrm{m}^{2}\right)$.

3.4. 30-Day Mortality. One patient in group L died due to malignoma-induced erosive bleeding of the pulmonary artery on the second day after surgery. One patient in group E died 28 days after surgery due to septic shock and severe mediastinitis. Thus, 30 -day mortality was $7.4 \%$ for all patients, $9.1 \%$ in group L, and $6.3 \%$ in group $\mathrm{E}$.

3.5. Metabolic Data. Lactate levels, central venous oxygen saturation $\left(\mathrm{ScvO}_{2}\right)$, base excess $(\mathrm{BE})$, and hemoglobin $(\mathrm{Hb})$ are given in Table 3. Lactate levels increased significantly at 6postop, 12postop, and 24postop in group $\mathrm{T}$ and group $\mathrm{E}$ compared to BL. However, levels of lactate remained very low $(<1.4 \mathrm{mmol} / \mathrm{L}) . \mathrm{ScvO}_{2}$ decreased significantly, in all groups at 12 postop, and 24postop compared to BL, but always remained in a range above $70 \%$. BE decreased significantly at 6postop, 12postop and 24postop compared to BL. Hb was also significantly reduced at most timepoints compared to BL. At this point it has to be clearly stated that all significant changes in all metabolic data were well within normal values and have to been seen clinically irrelevant.

In addition to EVLWI, $\mathrm{p}_{\mathrm{a}} \mathrm{O}_{2} / \mathrm{F}_{\mathrm{i}} \mathrm{O}_{2}$-ratio and $\mathrm{CI}$, further data on hemodynamics $\left(\mathrm{AP}_{\text {mean }}[\mathrm{mmHg}], \mathrm{CVP}\right.$ $[\mathrm{mmHg}]$, GEDI $\left.\left[\mathrm{mL} / \mathrm{m}^{2}\right]\right)$, SVI $\left[\mathrm{mL} / \mathrm{m}^{2}\right]$, pulmonary compliance $\left[\mathrm{L} / \mathrm{cmH}_{2} \mathrm{O}\right]$, and norepinephrine administration $[\mu \mathrm{g} / \mathrm{kg} / \mathrm{min}]$ are shown in Table 4 . In group L, 6 of 11 patients required norepinephrine administration temporarily; in group E, all patients required temporary norepinephrine administration.

\section{Discussion}

Although fluid management guided by functional parameters of preload are suggested to be rather fluid aggressive and validity of these parameters are controversial under open chest conditions, the present study shows that this goaldirected approach does not result in pulmonary fluid overload and deleterious reduction of pulmonary function in thoracic surgery requiring lateral thoracotomy and OLV. Furthermore, no derangement in metabolic parameters or increase in mortality associated with an altered pulmonary function could be identified. Whether this treatment strategy that demonstrated potential clinical benefit in abdominal and cardiac surgery is also potentially useful in this field 
TABle 3: Metabolic data. $\mathrm{ScvO}_{2}$ : central venous oxygen saturation; BE: base excess. ${ }^{*}$ Difference to BL in analysis of variance (ANOVA) $(P<0.05)$. BL: directly after induction of anesthesia; OLVimpl15: 15 minutes after beginning OLV; OLVterm15: 15 minutes after cessation of OLV; 6postop: 6 hours after surgery; 12postop: 12 hours after surgery; 24postop: 24 hours after surgery.

\begin{tabular}{|c|c|c|c|c|c|c|}
\hline & $\mathrm{BL}$ & OLVimpl15 & OLVterm15 & 6postop & 12postop & 24postop \\
\hline Lactate $_{\mathrm{T}}[\mathrm{mmol} / \mathrm{L}]$ & $0.8 \pm 0.3$ & $0.8 \pm 0.2$ & $1.1 \pm 0.5$ & $1.2 * \pm 0.6$ & $1.1^{*} \pm 0.4$ & $1.2 * \pm 0.4$ \\
\hline Lactate $_{\mathrm{L}}[\mathrm{mmol} / \mathrm{L}]$ & $0.8 \pm 0.4$ & $0.7 \pm 0.2$ & $1 \pm 0.5$ & $0.9 \pm 0.3$ & $1 \pm 0.4$ & $1.1 \pm 0.4$ \\
\hline Lactate $_{\mathrm{E}}[\mathrm{mmol} / \mathrm{L}]$ & $0.8 \pm 0.2$ & $0.9 \pm 0.2$ & $1.2 \pm 0.5$ & $1.4 \pm 0.6^{*}$ & $1.2 \pm 0.4^{*}$ & $1.2 \pm 0.3^{*}$ \\
\hline $\mathrm{ScvO}_{2 \mathrm{~T}}[\%]$ & $84.6 \pm 6.5$ & $85.7 \pm 5.6$ & $86.8 \pm 4.8$ & $76.9 \pm 4.9^{*}$ & $72.7 \pm 8.3^{*}$ & $72.6 \pm 9.0^{*}$ \\
\hline $\mathrm{ScvO}_{2 \mathrm{~L}}[\%]$ & $87.0 \pm 5.4$ & $87.3 \pm 4.9$ & $87.5 \pm 4.6$ & $76.8 \pm 4.9^{*}$ & $74.8 \pm 2.9^{*}$ & $74.0 \pm 6.7^{*}$ \\
\hline $\mathrm{ScvO}_{2 \mathrm{E}}[\%]$ & $82.9 \pm 6.7$ & $84.6 \pm 6.0$ & $86.3 \pm 5.1$ & $77.0 \pm 5.1$ & $71.7 \pm 10^{*}$ & $72.0 \pm 10.1^{*}$ \\
\hline $\mathrm{BE}_{\mathrm{T}}[\mathrm{mmol} / \mathrm{L}]$ & $-2.5 \pm 2.9$ & $-5.1 \pm 3.3^{*}$ & $-6.3 \pm 3.3^{*}$ & $-5.5 \pm 2.6^{*}$ & $-5.8 \pm 2.1^{*}$ & $-5.5 \pm 1.9^{*}$ \\
\hline $\mathrm{BE}_{\mathrm{L}}[\mathrm{mmol} / \mathrm{L}]$ & $-1.9 \pm 2.9$ & $-3.2 \pm 2.6^{*}$ & $-4.5 \pm 2.0^{*}$ & $-4.4 \pm 1.8^{*}$ & $-4.7 \pm 2.3^{*}$ & $-4.7 \pm 2^{*}$ \\
\hline $\mathrm{BE}_{\mathrm{E}}[\mathrm{mmol} / \mathrm{L}]$ & $-2.9 \pm 3.0$ & $-6.3 \pm 3.1^{*}$ & $-7.5 \pm 3.5^{*}$ & $-6.3 \pm 2.8^{*}$ & $-6.4 \pm 1.9^{*}$ & $-5.8 \pm 1.9$ \\
\hline $\mathrm{Hb}_{\mathrm{T}}[\mathrm{mg} / \mathrm{dL}]$ & $10.5 \pm 1.7$ & $9.1 \pm 1.9^{*}$ & $8.5 \pm 1.5^{*}$ & $9.0 \pm 1.5^{*}$ & $8.8 \pm 1.4^{*}$ & $8.7 \pm 1.4^{*}$ \\
\hline $\mathrm{Hb}_{\mathrm{L}}[\mathrm{mg} / \mathrm{dL}]$ & $11.0 \pm 1.8$ & $9.3 \pm 2.0^{*}$ & $8.8 \pm 1.8^{*}$ & $8.8 \pm 1.9^{*}$ & $8.4 \pm 2.0^{*}$ & $8.2 \pm 1.8$ \\
\hline $\mathrm{Hb}_{\mathrm{E}}[\mathrm{mg} / \mathrm{dL}]$ & $10.2 \pm 1.6$ & $9.0 \pm 1.8$ & $8.3 \pm 1.3^{*}$ & $9.2 \pm 1.2$ & $9.0 \pm 1.1^{*}$ & $9.0 \pm 1.0^{*}$ \\
\hline
\end{tabular}

of surgery cannot clearly be stated. However, incurring an increased risk of significant pulmonary fluid overload or critical reduction in pulmonary function seems not to be a clinically relevant problem.

In thoracic surgery, esophagectomy and lung surgery are counted amongst the most commonly performed surgical operations. In esophagectomy patients, clinical trials have demonstrated an increase in EVLWI $[29,30]$. Oshima and colleagues reported values of EVLWI $>10 \mathrm{~mL} / \mathrm{kg}$ perioperatively after esophagectomy without having a standardized protocol for fluid administration. Severe surgical trauma, lymphatic node extirpation, and systemic inflammatory response are seen as the main causes for this increase in EVLWI. Furthermore, OLV might also contribute to the development of a postoperative pulmonary edema, since it is known that OLV is correlated with pulmonary oxidative stress [21, 22]. In our study using SVV for guidance of fluid therapy, no significant increase of EVLWI in the subgroup of esophagectomy was found perioperatively and levels of EVLWI remained below $10 \mathrm{~mL} / \mathrm{kg}$.

In the patients that underwent lung surgery, EVLWI was even lower and did not exceed values of $9 \mathrm{~mL} / \mathrm{kg}$. However, the validity of EVLWI in lung surgery must be interpreted with caution, particularly if lung tissue is resected, as was the case in our study where pulmonary lobectomy and bilobectomy were performed. Basically, EVLWI is underestimated when lung tissue is resected because any decrease in pulmonary blood volume induced by lung tissue resection influences the intrathoracic blood volume. Since EVLWI is calculated as the difference between intrathoracic thermal volume and intrathoracic blood volume-which in this instance would be overestimated-EVLWI is underestimated following lung tissue resection $[31,32]$. Therefore, the results of EVLWI in the lung surgery group might be regarded as artificially low. Even if EVLWI was underestimated by $20 \%$, EVLWI would not exceed the maximum of $11 \mathrm{~mL} / \mathrm{kg}$ as seen in the lung surgery group (the highest value of measurement at OLVTERM15) and not $9 \mathrm{~mL} / \mathrm{kg} 24$ hours after surgery. This level is still within a clinically acceptable range when related to the results of Sakka and coworkers who described an EVLWI $>12 \mathrm{~mL} / \mathrm{kg}$ to be correlated with a worse outcome in critically ill patients [13].

Volume deficiency indicated by SVV was corrected using colloid infusion in our study. Crystalloid was administered only at a maintenance rate. Since it is known that, compared to colloids, only one fifth of the intravenously infused volume of crystalloids remains within the intravascular space, it must be assumed that a strategy based on a protocol using crystalloids exclusively would potentially have led to a higher EVLWI value and potentially a more pronounced deterioration in pulmonary function [33].

Gas exchange was reduced in the lung surgery group and in the esophagectomy group. In lung surgery, a decrease in the $\mathrm{p}_{\mathrm{a}} \mathrm{O}_{2} / \mathrm{F}_{\mathrm{i}} \mathrm{O}_{2}$-ratio can be explained by resection of lung tissue leading to a reduction of the alveolar surface necessary for gas exchange. In patients undergoing esophagectomy, postoperative deterioration of gas exchange (such as a decrease in the $\mathrm{p}_{\mathrm{a}} \mathrm{O}_{2} / \mathrm{F}_{\mathrm{i}} \mathrm{O}_{2}$-ratio) is common and clinically challenging [34-37]. However, the decrease in the $\mathrm{p}_{\mathrm{a}} \mathrm{O}_{2} / \mathrm{F}_{\mathrm{i}} \mathrm{O}_{2}$-ratio was only moderate and values always remained above $300 \mathrm{mmHg}$. Therefore, SVV-guided volume therapy seemed not to have aggravated this clinical problem.

After initiation of SVV-guided fluid management, CI was increased at two timepoints compared to the baseline measurement. Although the increase did not reach statistical significance at all timepoints, these data suggest that SVVguided fluid management contributes to an improved CI even in open chest thoracic surgery, being the basis for optimization of tissue oxygenation.

GEDI did not change significantly during the observation period. This fact provides evidence that the volume replacement strategy oriented to SVV led to a stable preload condition in these patients. A comparison group with more restrictive fluid management would have been desirable at this point and certainly, the lack of this comparison group remains the major limitation of this study. Other limitations have to be taken into consideration. Only the total amount of fluid administration 24 hours after surgery was recorded, and 
TABLE 4: Hemodynamic parameters and oxygenation. EVLWI: extravascular lung water index; CI: cardiac index; AP mean: mean arterial pressure; CVP: central venous pressure; GEDI: global enddiastolic volume index; SVI: stroke volume index; compliance: pulmonary compliance; NE: norepinephrine administration; * difference to BL in analysis of variance (ANOVA) $(P<0.05)$. BL: directly after induction of anesthesia; OLVimpl15: 15 minutes after beginning OLV; OLVterm15: 15 minutes after cessation of OLV; 6postop: 6 hours after surgery; 12postop: 12 hours after surgery; 24postop: 24 hours after surgery.

\begin{tabular}{|c|c|c|c|c|c|c|}
\hline & $\mathrm{BL}$ & OLVimpl15 & OLVterm 15 & 6postop & 12postop & 24postop \\
\hline $\mathrm{EVLWI}_{\mathrm{T}}[\mathrm{mL} / \mathrm{kg}]$ & $7.8 \pm 2.5$ & $8.4 \pm 3.9$ & $8.5 \pm 2.5$ & $8.2 \pm 2.9$ & $8.7 \pm 2.6$ & $8.1 \pm 2.4$ \\
\hline $\mathrm{EVLWI}_{\mathrm{L}}[\mathrm{mL} / \mathrm{kg}]$ & $7.9 \pm 1.7$ & $8.1 \pm 3.2$ & $8.5 \pm 2.5$ & $7.8 \pm 2.4$ & $8 \pm 1.8$ & $7.2 \pm 1.9$ \\
\hline EVLWI $_{\mathrm{E}}[\mathrm{mL} / \mathrm{kg}]$ & $7.8 \pm 3$ & $8.5 \pm 3.4$ & $8.6 \pm 2.6$ & $8.5 \pm 3.1$ & $8.98 \pm 3$ & $9.1 \pm 2.5$ \\
\hline $\mathrm{p}_{\mathrm{a}} \mathrm{O}_{2} / \mathrm{F}_{\mathrm{i}} \mathrm{O}_{2}$-ratio $\mathrm{T}[\mathrm{mmHg}]$ & $419 \pm 122$ & $186 \pm 94^{*}$ & $334 \pm 92 *$ & n.d. & n.d. & n.d. \\
\hline $\mathrm{p}_{\mathrm{a}} \mathrm{O}_{2} / \mathrm{F}_{\mathrm{i}} \mathrm{O}_{2}$-ratio $\mathrm{L}[\mathrm{mmHg}]$ & $462 \pm 140$ & $202 \pm 105^{*}$ & $338 \pm 112^{*}$ & n.d. & n.d. & n.d. \\
\hline $\mathrm{p}_{\mathrm{a}} \mathrm{O}_{2} / \mathrm{F}_{\mathrm{i}} \mathrm{O}_{2}-$ ratio $_{\mathrm{E}}[\mathrm{mmHg}]$ & $389 \pm 101$ & $174 \pm 87^{*}$ & $332 \pm 80$ & $330 \pm 111$ & $329 \pm 105$ & $303 \pm 74^{*}$ \\
\hline $\mathrm{CI}_{\mathrm{T}}\left[\mathrm{L} / \mathrm{min} / \mathrm{m}^{2}\right]$ & $2.8 \pm 0.9$ & $3.6 \pm 0.9^{*}$ & $3.5 \pm 0.8$ & $3.5 \pm 0.9^{*}$ & $3.6 \pm 0.9$ & $3.5 \pm 0.9$ \\
\hline $\mathrm{CI}_{\mathrm{L}}\left[\mathrm{L} / \mathrm{min} / \mathrm{m}^{2}\right]$ & $3 \pm 0.9$ & $3.4 \pm 0.66$ & $3.5 \pm 0.8$ & $3.6 \pm 1.1$ & $3.9 \pm 1.2$ & $3.6 \pm 1.2$ \\
\hline $\mathrm{CI}_{\mathrm{E}}\left[\mathrm{L} / \mathrm{min} / \mathrm{m}^{2}\right]$ & $2.7 \pm 0.9$ & $3.7 \pm 1^{*}$ & $3.5 \pm 0.7$ & $3.5 \pm 0.6$ & $3.5 \pm 0.7$ & $3.4 \pm 0.6$ \\
\hline AP mean $_{\mathrm{T}}[\mathrm{mmHg}]$ & $78.7 \pm 15$ & $74.9 \pm 14.1$ & $73 \pm 12.1$ & $78.9 \pm 14.1$ & $73.9 \pm 16.1$ & $76.6 \pm 8.2$ \\
\hline $\mathrm{AP} \operatorname{mean}_{\mathrm{L}}[\mathrm{mmHg}]$ & $84.8 \pm 13.3$ & $84.7 \pm 12.6$ & $80.1 \pm 14.1$ & $85.9 \pm 11.7$ & $81.3 \pm 14.2$ & $81.4 \pm 6.9$ \\
\hline AP mean $_{\mathrm{E}}[\mathrm{mmHg}]$ & $74.5 \pm 15$ & $68.2 \pm 11$ & $68.1 \pm 7.8$ & $74 \pm 13.7$ & $69.5 \pm 15.9$ & $73.8 \pm 7.7$ \\
\hline $\mathrm{CVP}_{\mathrm{T}}[\mathrm{mmHg}]$ & $7.4 \pm 2$ & $7.3 \pm 2.9$ & $6.6 \pm 2.7$ & $6.1 \pm 2$ & $5.8 \pm 2.4$ & $6 \pm 2.2$ \\
\hline $\mathrm{CVP}_{\mathrm{L}}[\mathrm{mmHg}]$ & $7.9 \pm 0.3$ & $7.2 \pm 2.5$ & $6.4 \pm 2.3$ & $5.9 \pm 2.2$ & $5.4 \pm 1.2$ & $5.5 \pm 1.5$ \\
\hline $\mathrm{CVP}_{\mathrm{E}}[\mathrm{mmHg}]$ & $7 \pm 2.6$ & $7.4 \pm 3.3$ & $6.8 \pm 3$ & $6.3 \pm 1.9$ & $6 \pm 2.9$ & $6.2 \pm 2.6$ \\
\hline $\mathrm{GEDI}_{\mathrm{T}}\left[\mathrm{mL} / \mathrm{m}^{2}\right]$ & $673 \pm 169$ & $642 \pm 152$ & $633 \pm 144$ & $649 \pm 114$ & $665 \pm 124$ & $658 \pm 123$ \\
\hline $\mathrm{GEDI}_{\mathrm{L}}\left[\mathrm{mL} / \mathrm{m}^{2}\right]$ & $702 \pm 167$ & $694 \pm 121$ & $689 \pm 170$ & $670 \pm 93$ & $657 \pm 138$ & $640 \pm 101$ \\
\hline $\mathrm{GEDI}_{\mathrm{E}}\left[\mathrm{mL} / \mathrm{m}^{2}\right]$ & $653 \pm 173$ & $607 \pm 165$ & $594 \pm 112$ & $635 \pm 128$ & $670 \pm 119$ & $669 \pm 137$ \\
\hline $\mathrm{SVI}_{\mathrm{T}}\left[\mathrm{mL} / \mathrm{m}^{2}\right]$ & $40.2 \pm 11.1$ & $48.4 \pm 12.4$ & $46.9 \pm 15.3$ & $49.6 \pm 13.6$ & $49.7 \pm 13.8$ & $47.3 \pm 13.9$ \\
\hline $\mathrm{SVI}_{\mathrm{E}}\left[\mathrm{mL} / \mathrm{m}^{2}\right]$ & $38.9 \pm 10.0$ & $50.2 \pm 13.7$ & $46.7 \pm 16.1$ & $47.8 \pm 12.3$ & $47.1 \pm 12.5$ & $46.5 \pm 12.8$ \\
\hline $\mathrm{SVI}_{\mathrm{L}}\left[\mathrm{mL} / \mathrm{m}^{2}\right]$ & $42.0 \pm 12.7$ & $46.1 \pm 10.4$ & $47.1 \pm 14.9$ & $52.0 \pm 15.5$ & $53.1 \pm 15.3$ & $48.5 \pm 15.8$ \\
\hline Compliance $_{\mathrm{T}}\left[\mathrm{L} / \mathrm{cmH}_{2} \mathrm{O}\right]$ & $0.049 \pm 0.013$ & $0.023 \pm 0.006^{*}$ & $0.046 \pm 0.012$ & n.d. & n.d. & n.d. \\
\hline Compliance $_{\mathrm{L}}\left[\mathrm{L} / \mathrm{cmH}_{2} \mathrm{O}\right]$ & $0.055 \pm 0.014$ & $0.024 \pm 0.006^{*}$ & $0.045 \pm 0.013$ & n.d. & n.d. & n.d. \\
\hline Compliance $_{\mathrm{E}}\left[\mathrm{L} / \mathrm{cmH}_{2} \mathrm{O}\right]$ & $0.046 \pm 0.01$ & $0.022 \pm 0.005^{*}$ & $0.046 \pm 0.012$ & $0.039 \pm 0.013$ & $0.052 \pm 0.023$ & $0.062 \pm 0.024$ \\
\hline $\mathrm{NE}_{\mathrm{T}}[\mu \mathrm{g} / \mathrm{kg} / \mathrm{min}]$ & $0.07 \pm 0.02$ & $0.12 \pm 0.02$ & $0.08 \pm 0.01$ & $0.04 \pm 0.02$ & $0.03 \pm 0.01$ & $0.02 * \pm 0.01$ \\
\hline $\mathrm{NE}_{\mathrm{L}}[\mu \mathrm{g} / \mathrm{kg} / \mathrm{min}]$ & $0.05 \pm 0.01$ & $0.09 \pm 0.02$ & $0.06 \pm 0.02$ & $0.01 \pm 0.01$ & $0.01 \pm 0.01$ & $0.008^{*} \pm 0.01$ \\
\hline $\mathrm{NE}_{\mathrm{E}}[\mu \mathrm{g} / \mathrm{kg} / \mathrm{min}]$ & $0.08 \pm 0.01$ & $0.14 \pm 0.01$ & $0.09 \pm 0.01$ & $0.06 \pm 0.02$ & $0.04 \pm 0.01$ & $0.03^{*} \pm 0.01$ \\
\hline
\end{tabular}

thus fluid administration cannot be differentiated according to the time line BL-24postop. The validity of SVV and the validity of transpulmonary thermodilution parameters during OLV have not been explored in detail. Thus far only one clinical study has shown SVV to be a predictor for volume responsiveness during OLV [38]. Furthermore, after thoracotomy with open chest conditions, SVV is not without controversy regarding prediction of volume responsiveness [23-28]. However, our data has revealed that even if SVVguided fluid management is not definitively validated under open chest conditions and OLV, severe pulmonary fluid overload is not inevitable.

Our study was not designed to demonstrate the clinical advantage of a SVV-guided fluid management in comparison to a control group. Furthermore, it is difficult to comment on any real safety in a study with a limited number of participants included, particularly when there is no comparison group. Therefore, our results have to be interpreted with caution. Nevertheless, our study forms the basis for further investigation regarding SVV-guided fluid management in thoracic surgery requiring open chest conditions and OLV, which has previously been effectively performed in other fields of surgery.

\section{Conflict of Interests}

Alwin E. Goetz and Daniel A. Reuter are members of the Medical Advisory Board of Pulsion Medical Systems. Apart from the conflict of interests mentioned above, all authors disclose (1) all funding sources, (2) any commercial or noncommercial affiliations, and (3) any other associations, such as consultancies.

\section{References}

[1] M. A. Hamilton, M. Cecconi, and A. Rhodes, "A systematic review and meta-analysis on the use of preemptive hemodynamic intervention to improve postoperative outcomes in moderate and high-risk surgical patients," Anesthesia and Analgesia, vol. 112, no. 6, pp. 1392-1402, 2011. 
[2] M. Buettner, W. Schummer, E. Huettemann, S. Schenke, N. Van Hout, and S. G. Sakka, "Influence of systolic-pressurevariation-guided intraoperative fluid management on organ function and oxygen transport," British Journal of Anaesthesia, vol. 101, no. 2, pp. 194-199, 2008.

[3] M. S. G. Goepfert, D. A. Reuter, D. Akyol, P. Lamm, E. Kilger, and A. E. Goetz, "Goal-directed fluid management reduces vasopressor and catecholamine use in cardiac surgery patients," Intensive Care Medicine, vol. 33, no. 1, pp. 96-103, 2007.

[4] J. Benes, I. Chytra, P. Altmann et al., "Intraoperative fluid optimization using stroke volume variation in high risk surgical patients: results of prospective randomized study," Critical Care, vol. 14, no. 3, article R118, 2010.

[5] M. R. Lopes, M. A. Oliveira, V. O. S. Pereira, I. P. B. Lemos, J. O. C. Auler Jr., and F. Michard, "Goal-directed fluid management based on pulse pressure variation monitoring during high-risk surgery: a pilot randomized controlled trial," Critical Care, vol. 11, article R100, 2007.

[6] M. Licker, M. de Perrot, A. Spiliopoulos et al., "Risk factors for acute lung injury after thoracic surgery for lung cancer," Anesthesia and Analgesia, vol. 97, no. 6, pp. 1558-1565, 2003.

[7] N. W. Brister, R. E. Barnette, V. Kim, and M. Keresztury, "Anesthetic considerations in candidates for lung volume reduction surgery," Proceedings of the American Thoracic Society, vol. 5, no. 4, pp. 432-437, 2008.

[8] M. Sentürk, "New concepts of the management of one-lung ventilation," Current Opinion in Anesthesiology, vol. 19, pp. 14, 2006.

[9] K. D. Eichenbaum and S. M. Neustein, "Acute lung injury after thoracic surgery," Journal of Cardiothoracic and Vascular Anesthesia, vol. 24, no. 4, pp. 681-690, 2010.

[10] J. Lemson, L. E. van Die, A. E. A. Hemelaar, and J. G. van der Hoeven, "Extravascular lung water index measurement in critically ill children does not correlate with a chest $\mathrm{x}$-ray score of pulmonary edema," Critical Care, vol. 14, no. 3, article R105, 2010.

[11] A. Laggner, G. Kleinberger, J. Haller, K. Lenz, G. Sommer, and W. Druml, "Bedside estimation of extravascular lung water in critically ill patients: comparison of the chest radiograph and the thermal dye technique," Intensive Care Medicine, vol. 10, no. 6, pp. 309-313, 1984.

[12] P. R. Eisenberg, A. S. Jaffe, and D. P. Schuster, "Clinical evaluation compared to pulmonary artery catheterization in the hemodynamic assessment of critically ill patients," Critical Care Medicine, vol. 12, no. 7, pp. 549-553, 1984.

[13] S. G. Sakka, M. Klein, K. Reinhart, and A. Meier-Hellmann, "Prognostic value of extravascular lung water in critically ill patients," Chest, vol. 122, no. 6, pp. 2080-2086, 2002.

[14] E. R. Johnson and M. A. Matthay, "Acute lung injury: epidemiology, pathogenesis, and treatment," Journal of Aerosol Medicine and Pulmonary Drug Delivery, vol. 23, no. 4, pp. 243 252, 2010.

[15] V. V. Kuzkov, M. Y. Kirov, M. A. Sovershaev et al., "Extravascular lung water determined with single transpulmonary thermodilution correlates with the severity of sepsis-induced acute lung injury," Critical Care Medicine, vol. 34, no. 6, pp. 16471653, 2006.

[16] G. S. Martin, S. Eaton, M. Mealer, and M. Moss, "Extravascular lung water in patients with severe sepsis: a prospective cohort study," Critical Care, vol. 9, no. 2, pp. R74-R82, 2005.

[17] J. Lohser, "Evidence-based Management of one-lung ventilation," Anesthesiology Clinics, vol. 26, no. 2, pp. 241-272, 2008.
[18] S. Haas, R. Kiefmann, V. Eichhorn, A. E. Goetz, and D. A. Reuter, "Hemodynamic monitoring in one-lung ventilation," Anaesthesist, vol. 58, no. 11, pp. 1085-1096, 2009.

[19] K. F. Cheong, "Re-expansion pulmonary oedema following one-lung ventilation-a case report," Annals of the Academy of Medicine Singapore, vol. 28, no. 4, pp. 572-573, 1999.

[20] Y. Asao, M. Kobayashi, N. Tsubaki, O. Kobayashi, and K. Uehara, "A case of re-expansion pulmonary edema after one lung ventilation for the radical operation of lung cancer," The Japanese Journal of Anesthesiology, vol. 52, no. 2, pp. 154-157, 2003.

[21] Y. J. Cheng, K. C. Chan, C. T. Chien, W. Z. Sun, and C. J. Lin, "Oxidative stress during 1-lung ventilation," Journal of Thoracic and Cardiovascular Surgery, vol. 132, no. 3, pp. 513-518, 2006.

[22] Y. Sugasawa, K. Yamaguchi, S. Kumakura et al., "The effect of one-lung ventilation upon pulmonary inflammatory responses during lung resection," Journal of Anesthesia, vol. 25, no. 2, pp. 170-177, 2011.

[23] D. A. Reuter, M. S. G. Goepfert, T. Goresch, M. Schmoeckel, E. Kilger, and A. E. Goetz, "Assessing fluid responsiveness during open chest conditions," British Journal of Anaesthesia, vol. 94, no. 3, pp. 318-323, 2005.

[24] D. A. Reuter, T. Goresch, M. S. G. Goepfert, S. M. Wildhirt, E. Kilger, and A. E. Goetz, "Effects of mid-line thoracotomy on the interaction between mechanical ventilation and cardiac filling during cardiac surgery," British Journal of Anaesthesia, vol. 92, no. 6, pp. 808-813, 2004.

[25] E. Lorne, Y. Mahjoub, E. Zogheib et al., "Influence of open chest conditions on pulse pressure variations," Annales Francaises d'Anesthesie et de Reanimation, vol. 30, no. 2, pp. 117$121,2011$.

[26] E. E. C. de Waal, S. Rex, C. L. J. J. Kruitwagen, C. J. Kalkman, and W. F. Buhre, "Dynamic preload indicators fail to predict fluid responsiveness in open-chest conditions," Critical Care Medicine, vol. 37, no. 2, pp. 510-515, 2009.

[27] P. A. H. Wyffels, P. Sergeant, and P. F. Wouters, "The value of pulse pressure and stroke volume variation as predictors of fluid responsiveness during open chest surgery," Anaesthesia, vol. 65, no. 7, pp. 704-709, 2010.

[28] M. Sander, C. D. Spies, K. Berger et al., "Prediction of volume response under open-chest conditions during coronary artery bypass surgery," Critical Care, vol. 11, no. 6, article R121, 2007.

[29] Y. Sato, S. Motoyama, K. Maruyama et al., "Extravascular lung water measured using single transpulmonary thermodilution reflects perioperative pulmonary edema induced by esophagectomy," European Surgical Research, vol. 39, no. 1, pp. 7-13, 2007.

[30] K. Oshima, F. Kunimoto, H. Hinohara et al., "Evaluation of respiratory status in patients after thoracic esophagectomy using PiCCO system," Annals of Thoracic and Cardiovascular Surgery, vol. 14, no. 5, pp. 283-288, 2008.

[31] R. Katzenelson, A. Perel, H. Berkenstadt et al., "Accuracy of transpulmonary thermodilution versus gravimetric measurement of extravascular lung water," Critical Care Medicine, vol. 32, no. 7, pp. 1550-1554, 2004.

[32] F. Michard, "Bedside assessment of extravascular lung water by dilution methods: temptations and pitfalls," Critical Care Medicine, vol. 35, no. 4, pp. 1186-1192, 2007.

[33] M. S. Strunden, K. Heckel, A. E. Goetz, and D. A. Reuter, "Perioperative fluid and volume management: physiological basis, tools and strategies," Annals of Intensive Care, vol. 1, article 2, 2011. 
[34] P. L. Byth and A. J. Mullens, "Peri-operative care for oesophagectomy patients," Australian Clinical Review, vol. 11, no. 1-2, pp. 45-50, 1991.

[35] T. A. Crozier, M. Sydow, J. R. Siewert, and U. Braun, "Postoperative pulmonary complication rate and long-term changes in respiratory function following esophagectomy with esophagogastrostomy," Acta Anaesthesiologica Scandinavica, vol. 36, no. 1, pp. 10-15, 1992.

[36] B. Z. Atkins and T. A. D’Amico, "Respiratory complications after esophagectomy," Thoracic Surgery Clinics, vol. 16, no. 1, pp. 35-48, 2006.

[37] M. Ikeguchi, M. Maeta, and N. Kaibara, "Respiratory function after esophagecotomy for patients with esophageal cancer," Hepato-Gastroenterology, vol. 49, no. 47, pp. 1284-1286, 2002.

[38] K. Suehiro and R. Okutani, "Stroke volume variation as a predictor of fluid responsiveness in patients undergoing one-lung ventilation," Journal of Cardiothoracic and Vascular Anesthesia, vol. 24, no. 5, pp. 772-775, 2010. 


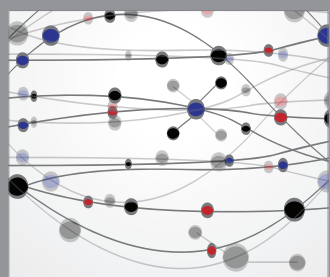

The Scientific World Journal
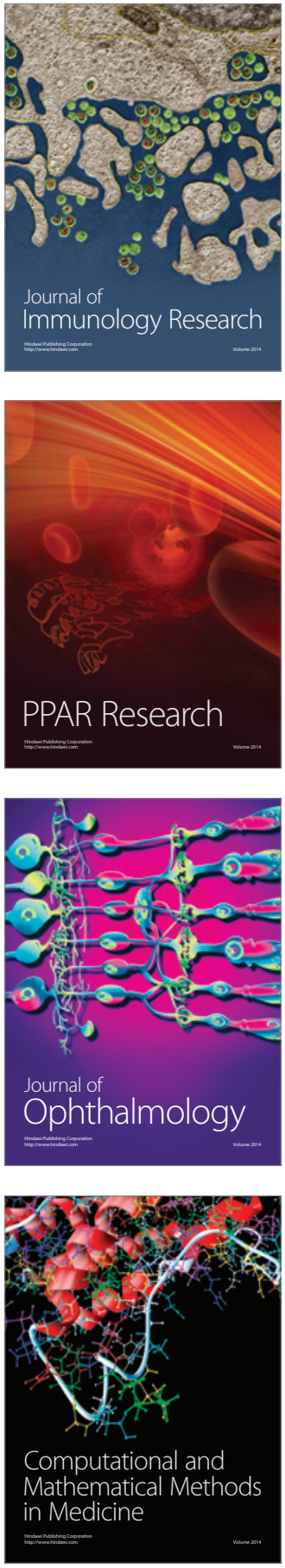

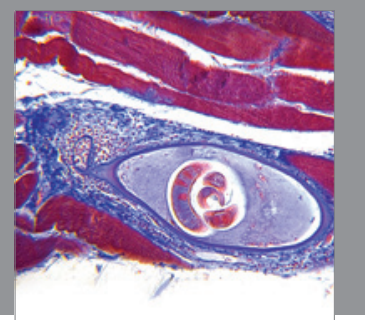

Gastroenterology

Research and Practice
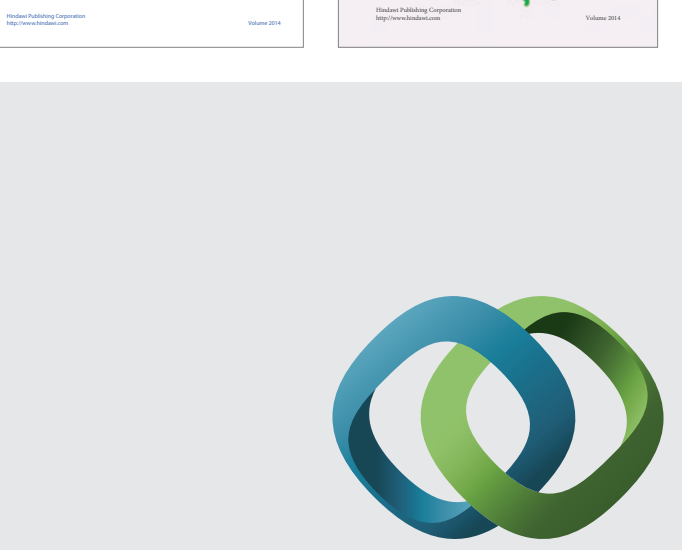

\section{Hindawi}

Submit your manuscripts at

http://www.hindawi.com
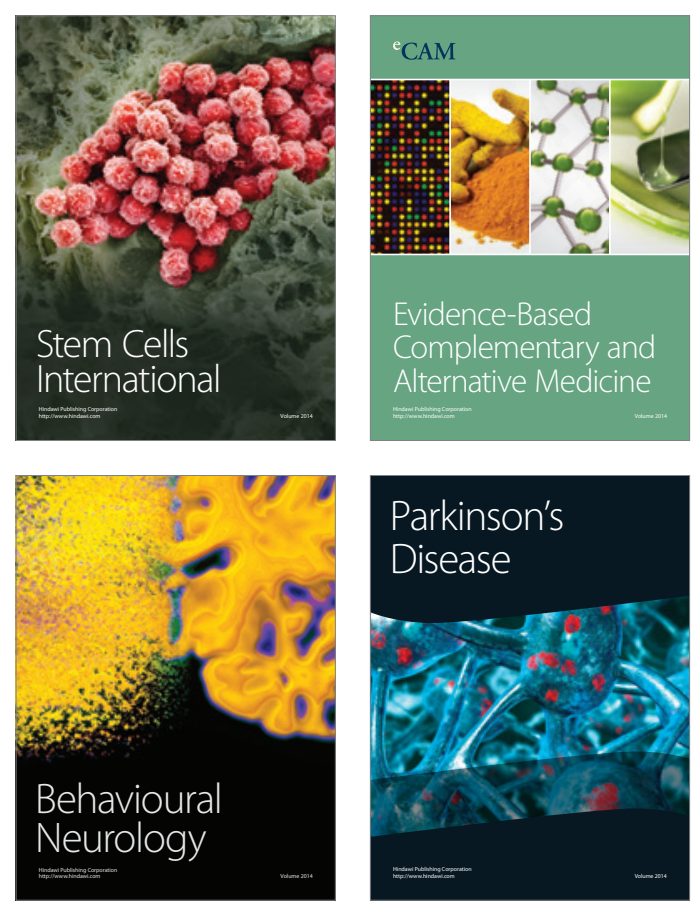

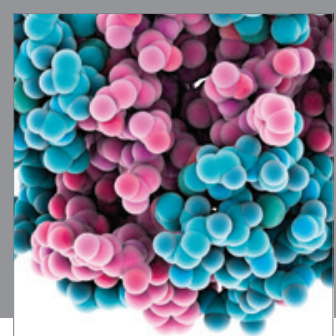

Journal of
Diabetes Research

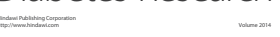

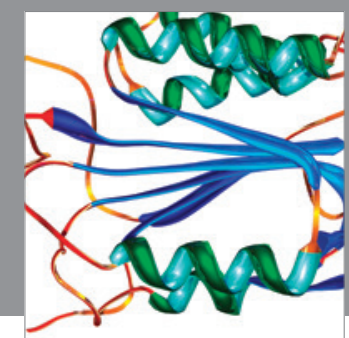

Disease Markers
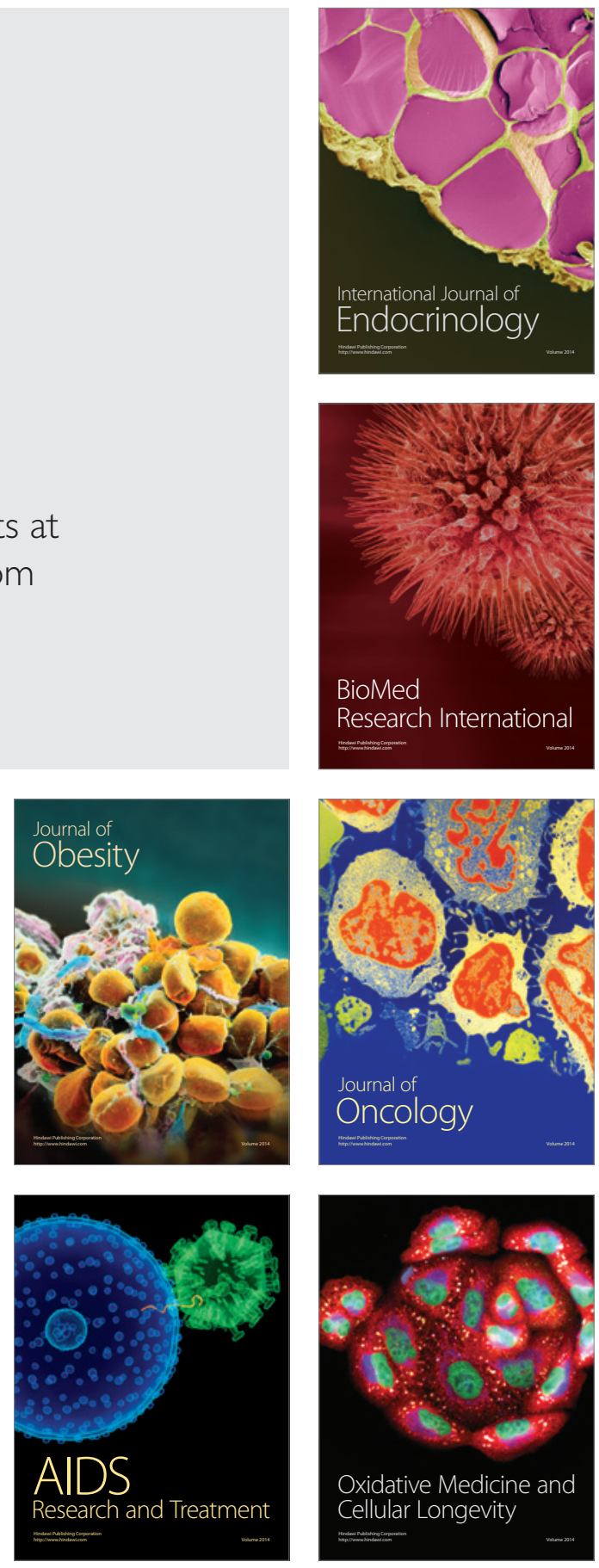